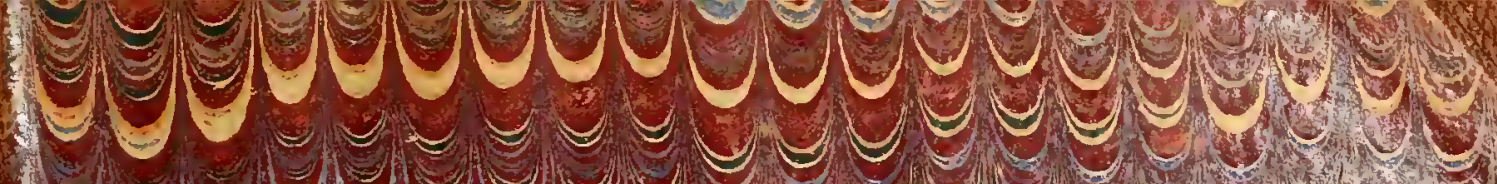

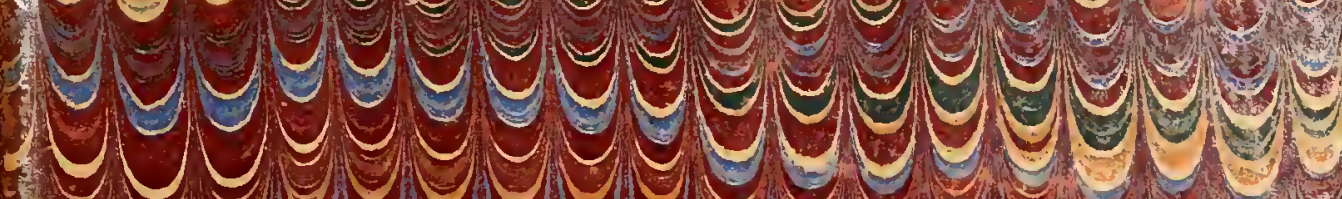

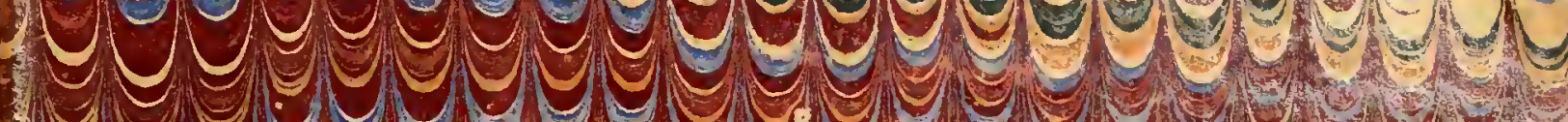
28

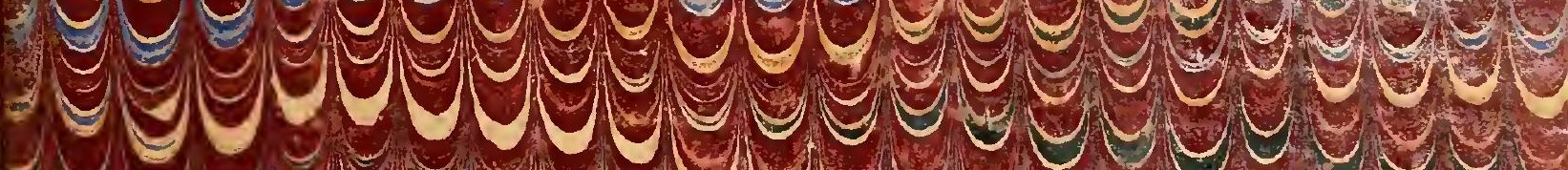

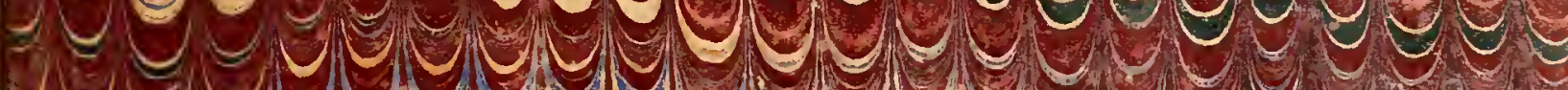

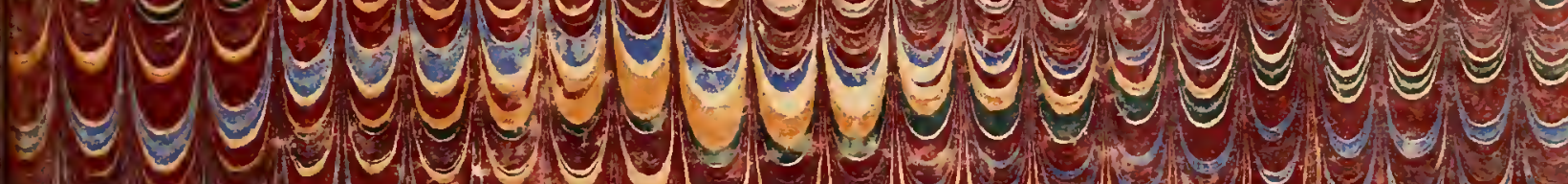
re y y y y

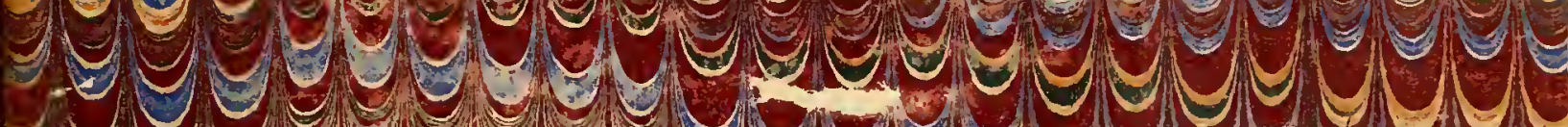

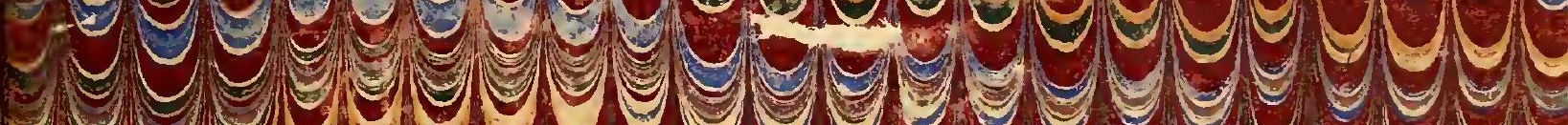

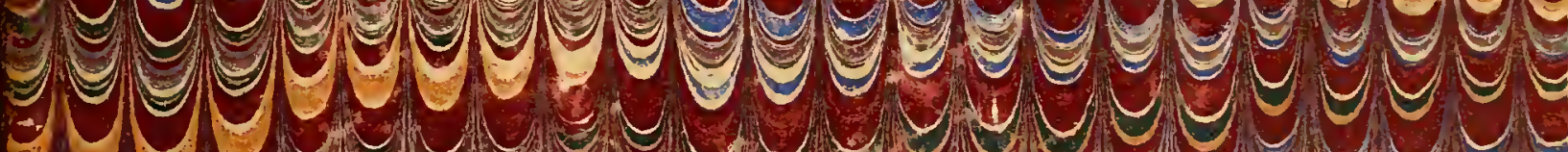
C - 69 19y S s s . 4 cos cos

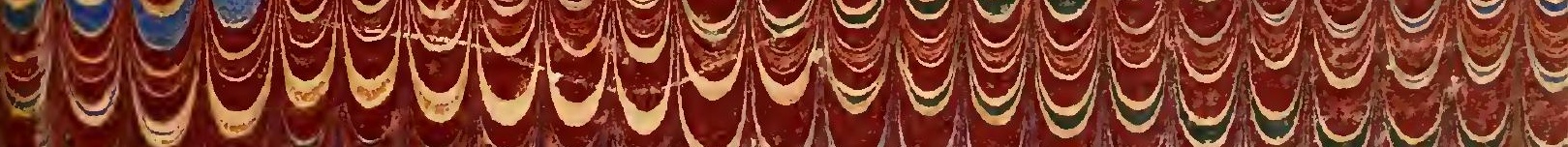
- 5 S a

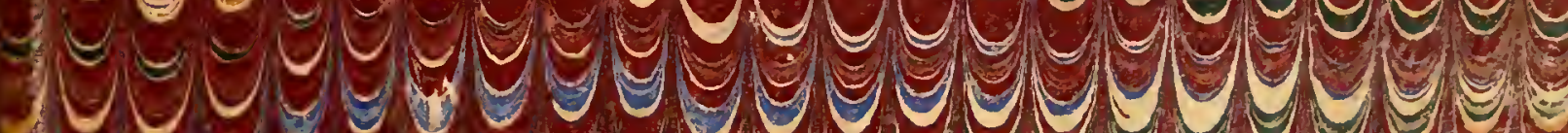

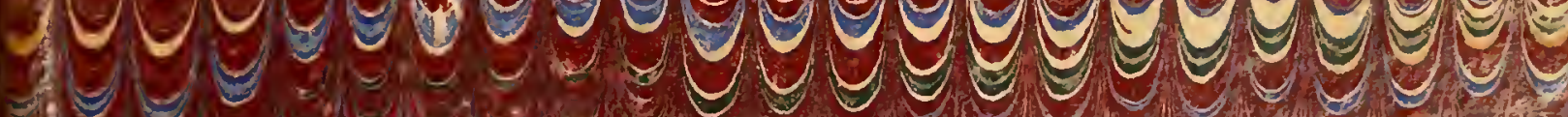

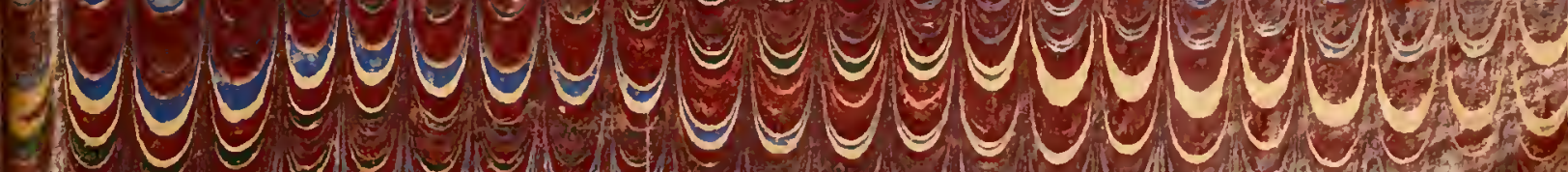
198 


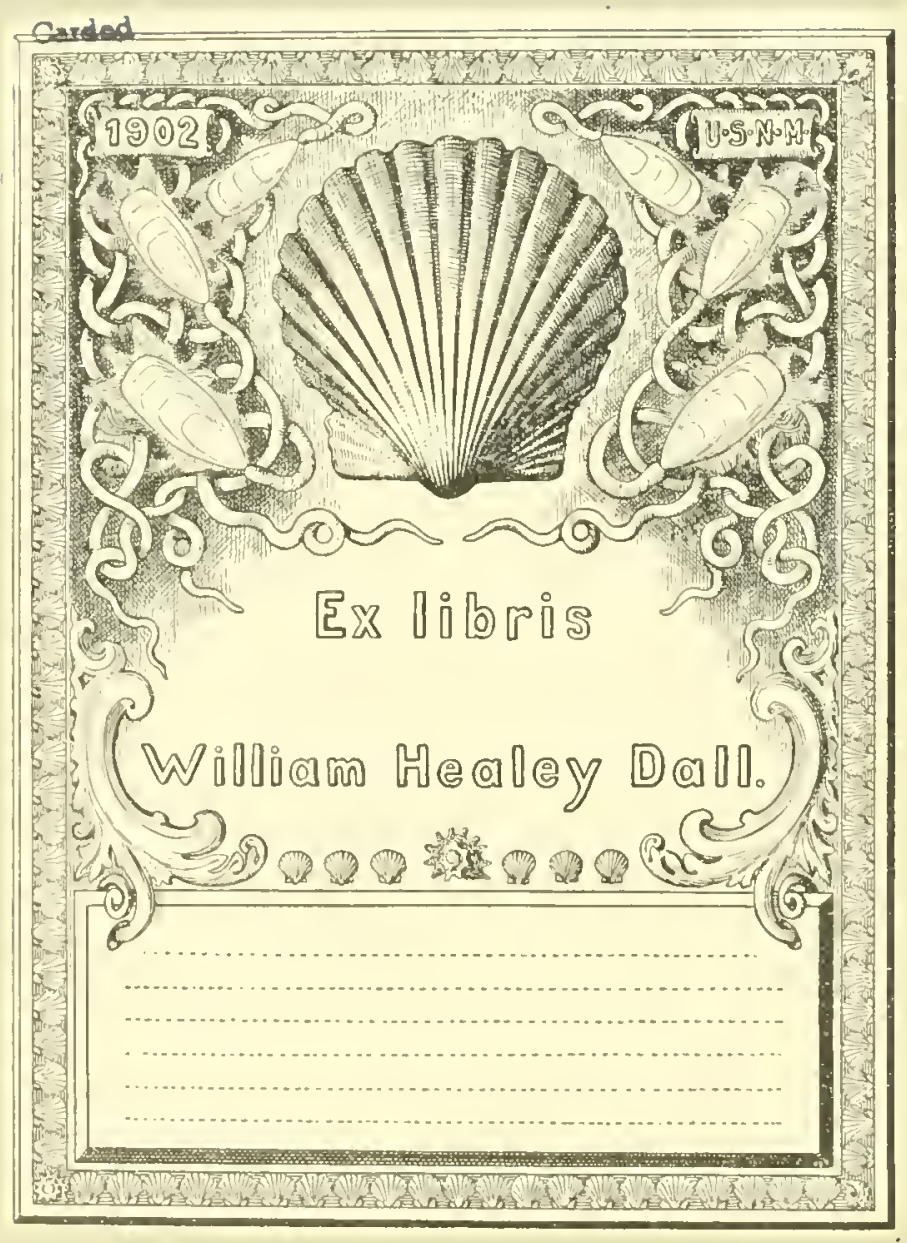


Divistion of Manly

Secticial La iry 



ESTRATTO DAL VOLUME II. DELLE MEMORIE

DELLA

SOGIETÁ ITALIANA DI SGIENZE NATURALI Tomo II, $\mathbf{~ I , ~} 9$.

\title{
PALEONTOLOGIA MALACOLOGICA
}

\section{DEI TERRENI TERZIARII DEL DISTRETTO DI MESSINA}

\author{
(PTEROPODI ED ETEROPODI)
}

DI

\section{GIUSEPPE SEGUENZA}

Professore reggente di Storia Naturale nel R. Liceo \& nell'Istituto Tecnico di Milessina, Socio di varie Accademie Scientifiche.

CON UNA TAVOLA

\section{MILANO}

COI TIPI DI GIUSEPPE BERNARDONI 


\section{PRESIDENZA PEL 1867}

Presidente, Dott. Emilo Connalia, Direttore del Museo Civieo di Milano, ecc., via del Monte Napoleone, 36.

Vice-Presidente, Antonio Villa, via della Sala, 3.

Dottor Giovann Omboni, Professore di Storia Naturale, via dei Cir-

Segretarj $\left\{\begin{array}{c}\text { co, } 12 . \\ \text { Abate Antonio Stoppani, Prof. di Geologia nel R. Istituto Tecnico }\end{array}\right.$ superiore in Milano, via di S. Maria alla Porta, 10.

Vice-Segretarj $\left\{\begin{array}{l}\text { Felice Franceschinr, via Broletto, } 16 . \\ \text { Camillo Marinoni, dottore in Scienze naturali, via S. Agnzese, } 5 .\end{array}\right.$ Cassiere, Giuseppe Gargantini Piatti, via del Senato, 14. 


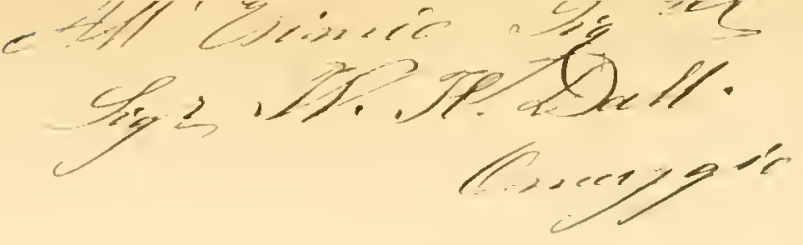

ESTRATTO DAL VOLUVE II. DELLE MEMORIE

DELLA

SOGIETÁ ITALIANA DI SGIENZE NATURALI

\title{
PALEONTOLOGIA MALACOLOGICA
}

\section{DEI TERRENI TERZIARII DEL DISTRETTO DI MESSINA}

\author{
(PTEROPODI ED ETEROPODI)
}

DI

\section{GIUSEPPE SEGUEYZA}

Professore reggente di Storia Natarale nel R. Liceo e nell'Istituto Tecnico di Mliessina,

Socio di varie Accademie Scientifiche.

\section{MILANO}

COI TIPI DI GIUSEPPE BERNARDONI 



\title{
PALEONTOLOGIA MALACOLOGICA
}

\section{DELLE ROGGE TERZIARIE DEL DISTRETTO DI MESSINA}

\author{
studiata nei suni rapporti zoologici, paleontologici e stratigrafici.
}

\section{PTEROPODI ED ETEROPODI}

\author{
PARTE PRTMA
}

\section{Plenopodi}

\author{
La zoologie bien applique doit elie le principal flambean de th \\ géologie................ \\ Deshayes. Anim. s. vert. du bassin de Paris, Introduction.
}

Se per poco volgiamo uno sguardo a quella parte del Mediterraneo che giace tria le coste orientali della Sicilia e le occidentali della Calabria, a quelle placide acque ehe bagnando le amene spiagge del littorale messinese, ed internandosi nel suo bel porto, costituiscono tanta deliziosa regione nel Mediterraneo, resteremo sommamente sorpresi nel vederle popolate d'immense e variate schiere d'animali pelagici, che succedendosi vicendevolmente a norma delle stagioni, ed apparendo alla superficie delle onde a norma delle ore stesse, hamno dato vastissimo campo ai zoologi d'intraprendere lunghe e serie ricerche, di aricchire la scienza di nnovi trovati, e i gabinetti di numerose e variate collezioni. Quivi infatti ai multiformi e deliziosi pesci si aggiungono i pelagici molluschi, l' elegante Argonauta, i diafani Eteropodi, gli agili Pteropodi; quivi le acque pullulano di variatissime Meduse che presentano i fatti piì strani della generazione alternante; quivi numerosi ed eleganti Vermi dai movimenti varii animano le onde; quivi nuotano le sociali e diafane Sarpe, ed immense colonie di Sifonofore fanno mostra dei loro svariati colori e dei loro multiformi individui; quivi col possente ainto del microseopio si osserva una miriade di elegantissimi Radiolarii, minimi protozoi dallo scheletro siliceo, che nella loro somma picciolezza hanno forme si varie ed eleganti, da fire ripetere con Linneo: Natura marime miranda in minimis.

Si numerosi e variati animali che popolano doviziosamente il mare di Messina, hamno attirato in ogni epoca nelle nostre regioni distintissimi zoologi di prossime e di lontane contrade ad esaminare le loro forme nello stato embrionario, nelle varic 
metamorfosi che subisce il loro corpo nella sua evoluzione, e nello stato adulto; « studiare i loro costumi e le loro anatomiche strutture, ad indagare la loro fisiologia, regalando quindi alla scienza sempre nuovi trovati e scoperte della più alta importanza. Lo dicono a chiare note le grandi scoperte del Miiller sulla embriogenia c le metamorfosi degli Echinodermi (1), e quell'altra di un nuovo ordine di Rizopodi, che egli nominava Radiolarii (2), e clie furono da Iui con tanta cura studiati nelle acque del porto di Messina, e poscia, con pazientissime ricerche, riesaminati dall'Heckel (3), che ne scopriva nel porto stesso numerose e variatissime specie; lo ripetono ad evidenza i tanti altri lavori del Philippi, del Cantraine, del Bènoit, del Keferstein, dello Stuart e di tanti altri valentissimi naturalisti.

Ma tra tanti organismi pelagici volgiamo le nostre considerazioni ai Molluschi P'teropodi, a quegli esseri malacologici che, alle espansioni aliformi, ai rapidi movimenti, alle conchiglie diafane, peculiarmente ed in varii modi conformate, ben si discernono da tutti quegli altri molluschi dai lenti e limitati movimenti, che or strisciano sul suolo, or immobili stanno immersi nei fondi limacciosi, ed or son condannati a star fissi alle rocec.

I Pteropodi admque nei erepuscoli primaverili appaiono a miriadi nelle acque di Messina, e non di raro occorre di vedere anco le sabbie della spiaggia ricoperte dalle loro spoglie che sono state rigettate dai marosi, e sovente le placide onde del porto danno agio allo studio dei loro costumi, delle loro funzioni e delle loro variate forme, che sono state in varii tempi esaminati da distinti zoologi.

Dopo le ricerche infatti del Cantraine (4), del Philippi (5), del Benoit (6) noi sappiamo che vivono nei nostri mari i seguenti Pteropodi:

$$
\begin{aligned}
& \text { Hyalaea tridentata Lamk. } \\
& " \quad \text { gibbosa Rang. } \\
& " \quad \text { Melly Benoit. } \\
& " \quad \text { vaginella Cantraine. } \\
& \text { Diacria trispinosa Lesueur. } \\
& \text { Cleodora lanceolata Per. e Lesueur. } \\
& \text { " cuspidata Quoy c Gaym. } \\
& \text { Creseis spinifera Rang. } \\
& \text { " acicula Rang. } \\
& \text { " striata Rang. }
\end{aligned}
$$

delle quali talune vi compariscono sovente con grande profusione, formando immense societa di mollusehi pelarici.

(1) I. MüLler. Ueber den allgemeinen Plan in der Entwieldung Echinodermen. (Memor. dell'Accademia di Berlino).

(2) Mülcer. Monatsberiet. d. Akad. 1855, 1856 e 1858. Mem. de la Academie des sciences de Berlin, 1858.
(3) E. Heckel. Dic Radialarien (Rhizopoda radiaria). Berlino, 1862.

(4) Malacologie méditerranéenne. Bruxelles, 1840.

(5) Enumeratio molluscorum utriusque Siciliae, $1836-44$.

(6) Ricerche malacologiche. Messina, 1843. 
Or le peregrinazioni geologiche che da parechi anni formano le mie ordinarie distrazioni nelle poche ore libere dalle svariate e molteplici occupazioni di officio e di professione, mi hanno fatto apprendere di buon'ora che i mari terziarii di Messina non abboudavano meno di Pteropodi di quanto ne sono riechi quelli dell' epoea attuale.

Sin da lungo tempo infatti io seopriva le tracce di tali molluschi in tutti gli strati terziarii del Messinese, ed in talmne contrade osservava abbondanti residui delle loro conchiglie che attestano l'abbondanza di tali esseri viventi in quelle epoche. Alla eontrada Trapani presso Messina osservavo taluni strati di marna giallastra e grigiastra, in cui le Hyalaea e le Cleodora sono sparse a grande dovizia; nei grandi banclii di calcare pliocenico presso S. Filippo, tra l'immenso accumulo di Brachiopodi, bene spesso si raccolgono abbondantemente le Cleodore, sebbene mal eonservate e rotte. Nella primavera dello scorso anno, portando le mie indagini in una eava di argilla miocenica presso la eontrada Bianchi, vi scopriva uno strato di argilla bruna colorata da acerdesio terroso in cui, tra i varii fossili, a grande profusione eravi sparsa una Hyalaea, ed in qualunque luogo esploravasi quello strato, bisognava romperlo a norma della sua strutturi schistosa, e ciascuma lamina irregolare della roecia, lasciava vedere numerosi individui della Hyalaea accennata, variamente deformati e compressi. Nel corso di questo anno poi, uel ealcare F presso Valdina, mi occorreva osservare, di unita agli alumni liceali, grande abbondanza di Jalee e di Cleodore, miste a Terebratule, a Coralli, a residui varii di Gasteropodi e di Lamellibranchiati. Erami noto d'altroncle che a Pagliarino, Scirpi, Gravitelli, ed in molti altri luoghi, un tale strato va spesso fornito dei residui di siffatti mollusehi pelagici; che le marne soprastanti a Rometta, Scoppo, Gravitelli ed altrove, oltre le Jalee e le Cleodore, racchiudono la conchiglia di una Spirialis; e eonoscevo benanco sin da molto tempo che le sabbie pleistoceniche racchiudono qualche residuo di conchiglie somiglianti.

Se intanto si mette in considerazione la estrema fragilitì di siffatte conclighlie, per la quale sono di continno esposte a venir distrutte faeilmente, e dall'azione corrosiva delle onde, eoadiuvata da quella delle sabbie e ghiaie delle spiagge sulle quali vengono d'ordinario rigettate, e da tante altre cagioni, si comprenderì agevolusente clue pochi sono queg'l individui che, resistendo alla distruzione, pervengono a formar parte dei sedimenti sottomarini; e quindi gli strati sedimentarii non possono racchindere che pochi residui delle varie specie che vissero nei mari delle diverse epoche geologiche.

Per tali considerazioni ragion vuole che copiosissimi Pteropodi fossero vissuti nei mari messinesi dell' epoche mioeniea, plioenica e pleistoeeniea, perehù potessimo oggigiono rinvenire tanti residui di questi molluschi, quanti ne abbiamo raccolto nelle rocce ealearee, argillose e marnose ele si deponevano in fondo ai mari di quelle epoche.

Tra i varii scrittori clie hanno trattato dei fossili delle rocce messinesi, solamente il Cantraine e l'Aradas accennano a residui di Pteropodi: il primo ri ha raccolto la Diacria trispinose Lesueur e la Cleodora lanceolata Per. e Lesuem, il secondo ha rinvenuto presso Gravitelli un sol modello di Cleodora, che riferi alla Cl. lanceolata; 
ma per quanto mi sappia nè il Philippi nè scrittori più moderni si sono imbattuti nei considerevoli depositi di Pteropodi che ho incontrato sì sovente nelle mie escursioni geologiche, e che mi lianno dato occasione di accennare nelle mie Notizie succinte intorno alla costituzione geologica dei terreni terziarii del distretto di Messina, la Hyalaea tridentata? Lamk., la Hyalaea trispinosa Les., la Cleodora lanceolata P. e L., e la Spirialis stenogyra (Scaea) Phil.

Le ulteriori ricerehe avendomi somministrato abbondanti materiali per lo studio di tali molluschi, mi hanno messo in grado di risolvere $\mathrm{i}$ dubbii insortimi riguarde alla determinazione di aleune specie, come lo addimostrano i segni d'interrogazione coi quali fui obbligato segnarne talune nelle precedenti pubblicazioni, e mi hanno messo in circostanze di descrivere talune specie nuove, portando a nove il numero totale, che era sinora di sole quattro, formando cosi un'altra monografia da aggiungersi alla mia Paleontologia malacologica delle rocce terziarie messinesi.

\title{
CLASSE. - PTEROP0DI (Pteropoda) Cuvier 1804.
}

\author{
RAMIGLLA. - JALIDI (Ityalidee) D'Orbigny 1837.
}

Gen. Hyalra Lamk. 1799.

\section{Sp. Hyalaea penafinis Seg.}

Fig. $5 a, 5 b, 5 c$.

Sinonimia.

1862. 'It. tridentata? Seguenza. Notizie succinte, ecc., pag. 18 (non Lamarck.).

Caratteri. - II. testa inflata, tenui, subtiliter concentrice striata, inferne convexa gibbosissima, striisque distinctioribus signata, superne parno convexa, quinquecostata, costis prominentibus, convexiusculis, bene distinctis; lateribus profinde fissis; mucronibus tribus, lateralibus fere angubum rectum formantibus, medio longiore, cuspidato, curviusculo; apertura angusta, labro inferiore maxime incurvato; Iabro dorsali producto, lato, concheeformi, reflexo, lateraliter plica unica utrinque instructo.

Conchiglia allungata, tenuissima e molto rigonfia; colla superficie ornata da sottili strie concentriche, le quali sono piu distinte sulla giblosa regione ventrale presso l'apertura; il dorso è poco convesso e presenta cinque costole ben rilevate e distinte, delle quali la media è un po' più larga delle altre; ai lati la conchiglia presenta delle fenditure che si estendono sino alla regione posteriore, dove sono tre speroni, dei quali i due laterali sono costituiti dall' incontro quasi ad angolo retto dei margini laterali col margine posteriore, quello mediano è molto pii lungo, in forma di aculeo alquanto curvo; l'apertura è molto strettil, il labbro inferiore è fortemente currato in dentro, e pereiò molto convesso; il labbro dorsale ì sporgente, largo, concavo, incurvo o presenta ai lati due pieghe brevi e rotondate. 
Rapporti e differenze. - Non posso trascurare dal manifestare qui i miei dubbii in riguardo alla definizione della specie or ora descritta; essa è talmente somigliante alla $H$. tridentata Lamk., che lascia molta incertezza intorno alla sua disgiunzione da quella; ma siccome essa è un po' più allungata, e perciò proporzionalmente piì stretta, colle costole molto rilevate e ben distinte, a differenza della $U$. tridentata, nella quale sono poco discernibili, col labbro dorsale più largo e segnato da due pie. ghe laterali; ed inoltre siccome cosifatti caratteri si conservano costautemente in tutti gli esemplari da me esaminati, ho creduto che le suddette differenze sieno valevoli a giustificare la disgimzione della nostra specie fossile dalla $H$. tridentata vivente.

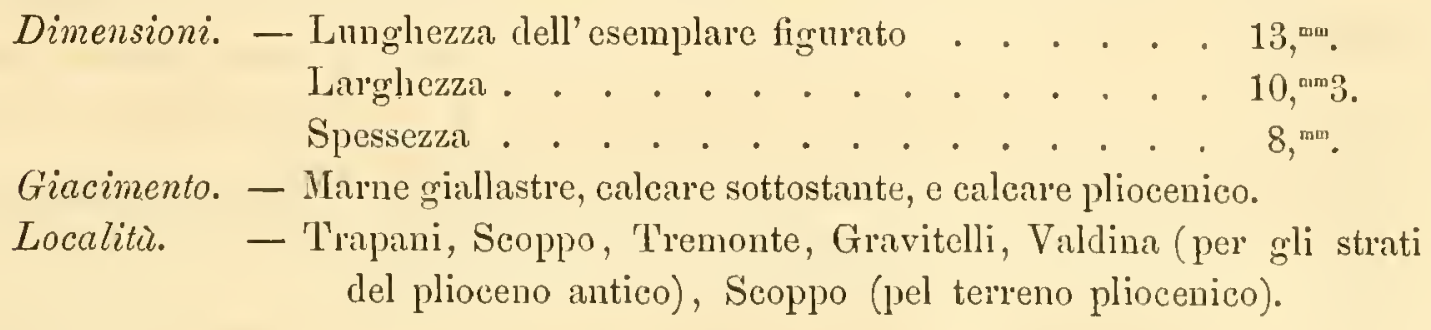

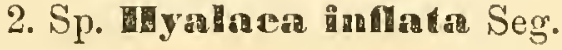

Fig. $6 a, 6 b, 6 c$.

Caratteri. - H. testa uirinque inflata, temu; inferne regulariter convexa gibbosissima; superne medio gibbosa ad latera planata, subcequaliter quinquecostata, costis prominentibus rotundatis interstitia suboquantibus, lateribus profunde fissis, marginibus lateralibus postea subrectis, cum posteriore ad conjunctionem angulis rectis formantibus, mucrone posteriore parro; apertura angusta, labro inferiore maxime incurvato, labro dorsali producto, rotundato, stricto, concavo, reflexo.

Conchiglia rigonfia molto, sottilissima, convessa regolarmente alla parte inferiore, alla regione dorsale convessa nel centro ed appianata sui lati; con cinque costole rotondate e molto rilevate, quasi eguali, e tanto larghe quanto gl'interstizii; ai lati sono due fenditure che si estendono sino alla regione posteriore, la quale presenta un margine retto, che riunenrosi ai margini laterali forma due angoli retti, e nel centro porta un piccolo sperone, cle trovasi sempre mutilato.

Rapporti e differenze. - La specie testè descritta è distinta eminentemente per la sua grande convessiti sulla regione dorsale, la quale d'ordinario suole essere quasi appianata nelle rarie specie. Una tale gibbosità dorsale vedesi anche nella $H$. taurineresis Sisu., che poi per tutt'altri particolari d̀ diversissima dalla mia specie.

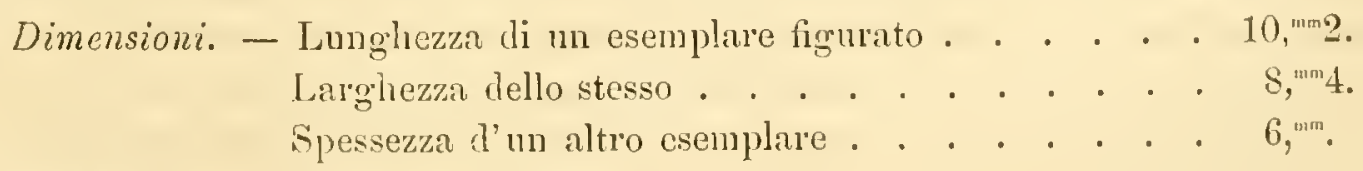

Giacimento. - Argille mioceniche.

Localita. - - Monforte, Biunchi. 
Sotto-genere Diacria Gray 1840.

3. Sp. Diacria tarispinosa Les.

Fig. $7 a, 7 b$.

Sinonimia.

1780-95. . . . . Chemn. VIII, vig. 13, lit. F. et lit. $a, b, c$.

1816-30. H. trispinosa ... . Lesuenr apud Blainville (Dict. des sc. nat. XXII, p. 82).

1827. "mucronata.... Quoy e Gaim. Anı. Sc. nat. X, pag. 231, f. 8.

1831. "triacantha.... Guidotti. Bromn. Ital., pag. 85.

1832. "depressa..... Bivona. (Nelle Effemeridi scient. e lett. per la Sicilia).

1832-35. "trispinosa ... Q Quoy e Gaim. Astr., II. p. 378, tav. 27, fig. 17 e 19.

1835-42. "mucronata.... D'Orb. Voy. dans l'Am., tom. 7, fig. 6-10.

1835-42. "trispinosa ... " " tom. 7, fig. 1-5.

1835-43. " $\quad \ldots$ Lamk. $2 .^{3}$ ediz. VII, pag. 417, 11. 4, 421, n. 15.

1836. " depressa .... . Phil. Enum. moll., vol. I, pag. 101.

1840. "trispinosa ... . Cantraine. Malac. med., pag. 28, tav. I, fig. 4.

1843. "depressa.... Benoit. Ricerche malacol., pag. 4, tav. I, fig. 5 a', $b$.

1844. "trispinosa ... . Phil. Enum. moll., vol. II, pag. 71.

1855. " depressa .... . Pictet. Traité de paléontologie, tom. III, pag. 318.

1859. Diacria trispinosa. Gray (Chenu. Man. de Conch., t. I, p. 109, fig. 465-466).

1862. H. trispinosa. . . G. Gegnenza. Notizie succinte, ecc., pag. 18 e 24.

1864. $" \quad \ldots$ A. Conti. Il monte Mario ed i snoi fossili, pag. 26.

Caratteri. - D. testa compressa, tenui, subtriangulari, vix striata; valva' dorsali quinque-costata; ventrali lateraliter unicostata, cuspidibus tribus longissimis, rectis; lateralibus ad axin testae perpendicularibus. Apertura angusta; labiis brevibus, vix. inflexis. (Cantraine).

Conchiglia compressa e molto fragile perchè assai sottile, di forma quasi triangolare ed appena striata; le due lamine che la costitniscono sono presso a poco ugualmente convesse; la dorsale presenta cinque costole, delle quali le due piì esterne alquanto pii larghe, la ventrale ha ma costola sopra ciascun lato; alla parte posteriore la conchiglia è fornita di tre speroni molto lunghi e retti, dei quali i clue laterali sono perpendicolari all' asse; l'apertura è stretta pel ravvicinamento dei labbri, che sono brevi, poco incurvati, ed alquanto disuguali, essendo il dorsale un po' piì sporgente. 
Rapporti e differenze. - Questa specie, per le sue lamine poco convesse, e pei speroni, e talmente distinta dalle Jalee tutte, che costitnisce pei moderni un gruppo a sè, il quale era stato elevato dal sig. Gray al rango di genere.

Giacimento. - Vivente nel Mediterraneo ed a Cuba.

Fossile nelle rocee plioceniche, Piacentino e Sienese (Cantraine) Monte Mario (A. Conti), Palermo (Philippi, Cantraine), Capo Peloro (Cantraine).

Nelle rocce messinesi raccogliesi raramente nel calcare $\mathrm{F}$ e nelle marne giallastre soprastanti, e nel calcare pliocenico.

Localita. - Pagliarino, Valdina (nel calcare), Scoppo, Trapani (nelle marne), Scoppo, S. Filippo (nel calc. pliocen.).

\section{Genere Cleodora P. e L. 1810.}

\section{Sp. Cleorlore Ianceolata P. L.}

Fig. $8 a, 8 b, 8 c$.

Sinonimia.

Cleodora pyramidata. Peron. Ann. XV, tav. 2, n. 14.

$" \quad$ lanceolata. Lesneur. Nouv. Bull. III, n. 69, tav. 5, fig. 3.

1815-22. Cleodora pyramidata. Lamk. VI, 1. ${ }^{\text {a }}$ pag. 290.

1816-30. " " De Blainville. Dict. XXII, pag. S0, tav. 64.

1832-35. " " Quoy e Gaym. Voy. de l'Astrol. II, p. 386, tav. 27, fig. 7-13.

1835-43. " " Lamk. VII, 2., pag. 429 .

1835-42. $" \quad$ D'Orbigny. Voy., p. 113, tav. 7, fig. 25-32 e tav. 8, fig. $32-33$.

1836. " lanceolata. . Seacchi. Catal., pağ. 18.

1836. " " Philippi. Enmmerat. moll., ecc., pag. 102, vol. I.

1840. Hyalaea pyramidata. Cantraine. Malac. med., pag. 30, tav. I, fig. 9.

1843. Cleodora lanceolata. Benoit. Ricerch. malacolog., p. 5, tav. 1, fig. 6 a, 6.

1844.

1847.

1855 .

$\begin{array}{ll}n & n \\ n & n\end{array}$

1859.

1862.

1864.
Philippi. Enum,, vol. II, pag. 71.

A. Aradas. Fossili di Gravitelli, pag. 15.

Pictet. Traité de paléontologie, tom. III, pag. 319, tav. LXX, fig. 19.

Chenu. Man. de conch., vol. I, pag. 109, fig. 467.

? G. Seguenza. Notizie succinte, ecc., pag. 18, 24 e 30.

Conti. Monte Mario, pag. 26.

Caratteri. - C. testa compressa, rhomboidali, leviter arcuata, hyalina, striata striis undulatis; valva dorsali in medio obtuse carinata, producta, lateraliterque radiatim costulata, ventrali concavo-plana, in medio unicostata; cuspide basilari subrecta (Cantraine). 
I molti esemplari di Cleodora che ho raccolto nelle rocce messinesi, essendo più o meno rotti e malconservati, non mi permettono di dare estesa e compiuta descrizione, ma mi lasciano purnondimeno sicuro della detcrminazione specifica. Infatti, comparati questi cogli esemplari viventi della Cleodora lanceolata, corrispondono con essa precisamente ed in tutti i particolari. La forma, la carena e le costole dorsali, la costola mediana della regione ventrale sono identicissime.

L'esemplare rappresentato dalla fignra $8 c$ è un po' diverso dagli altri per le strie traspersali abbastanza distinte che ornano la costola della valva ventrale, ma tal carattere non costituisce che una variazione del tipo normale.

Rapporti e differenze. - Questa specic è ben distinta dalle altre per la sua forma romboidale, e molto clargata como la $C$. cuspidata, la quale si distingue principalmente per le sue tre lunghe prominenze aciculatc, rette, divergenti e dirette nel scnso dell' apertura.

Dimensioni. - - Il cattivo stato dei nostri esemplari fossili non mi permette di riportare qui le esatte dimensioni, purnondimeno posso asserire che la loro grandezza è presso a poco identica a quella clegl'individui viventi.

Giacimento. - Vivente nel Miediterranco.

Fossile nelle rocee plioceniche, Capo Pcloro e Piacentino (Cantraine), Palermo e Monteleone (Philippi), Monte Mario (Conti) ecc., Messina (G. Seguenza).

$\mathrm{Nel}$ Messinese raccogliesi nel calcare $\mathrm{F}$, nelle marne gialle $\mathrm{E}$, nel calcare pliocenico soprastante e nelle sabbie quaternarie.

Località. - Nel calcare F. Valdina, Pagliarino. Nelle marne Rometta, Trapani, Scoppo, Gravitelli, ecc.

Nel calcare pliocenico Gravitelli, S. Filippo e Rometta. Nel quaternario Milazzo e Gravitelli.

\section{Sp. Cleodlora cuspidata Quoy e Gaym.}

$$
\text { Fig. } 10 a, 10 b \text {. }
$$

Sinonimia.

Cl. cuspidata. . . . Bosq. Coq. II, pag. 238, tav. 9, fig. 5-7.

1816-30. " " ... Blainville. Diction., ecc., XV, tav. 2, fig. 35.

1832-35. " $"$.... Quoy e Gaym. Voy. de Astrol. Moll., tom. 2, pag. 238, tav. 27 , fig. $1-5$.

1835-42. $\quad " \quad \ldots$ D'Orbigny. Voy., pag. 112, tav. 7, f. $20-24$.

1835-43. " " ... Lamarck. 2..$^{a}$ odiz. VII, pag. 416.

1840. IIyalaea cuspidata. Cantraine. Malacol. med., pag. 29, tav. I, fig. 8.

1844. Cl. cuspidata. . . Plilippi. Enumeratio moll., pag. 71, vol. II.

1859. " " ... Chenu. Manuel de Conclı,, vol. I, pag. 109, fig. 469.

Caratteri. - C. testa compressa, trigona aut subrhomboidali, hyalina, fragilissima profunde fissa; valva dorsali medio carinaia, lateraliter radiatim costulata, 
concentrice striato-sulcata; sulcis undulatis; valva ventrali striata, medio convexa, carinato-rotundata; apertura transversa, subquadrangulari; cuspidibus quatuor quarum prima basilari, brevi, adunca; cateris tribus rectis, divergentibus, gracilibus, quarum lateralibus canaliculatis; tertia in medio labro dorsali sita. (Cantraine).

L'unico esemplare che possiedo è il frammento figurato nell' annessa tavola; esso, comparato ai viventi dei nostri mari, mostra con essi un accordo compiuto in tutti $\mathrm{i}$ caratteri; infatti la carena dorsale molto elevata, le costole laterali, la regione ventrale colla sua costola mediana sporgente e rotondata, e coi lati segnati da solelii o pieghe trasversali sono conformi ai caratteri che distinguono la conchiglia della C. cuspidata vivente (1).

Rapporti c differenze. - Questa specic è distintissima da tntte le altre conosciute per essere molto dilatata, fortemente carenata, e sopratutto per le tre lunghe spine divergenti, che hanno origine dagli angoli dell'apertura.

Dimensioni. - La lunghezza del nostro frammento è di $8^{\text {mа }}$.

Giacimento. - La specie di che discorro conoscesi sinora allo stato vivente nell'Atlantico e nel Mediterraneo.

L' esemplare fossile da me posseduto proviene dal calcare pliocenico.

Localitic. - S. Filippo inferiore presso Messina.

\section{6. ${ }^{\text {S }}$ Sp. Cleodora trigoma. Seg.}

Fig. $9 a, 9 b$.

Caratteri. - C. testa compressiuscula elongata, recta, transvcrsim striata, striis rectis; valva dorsali in medio cxacte carinata, producta, lateraliterque radiatim costulata; ventrali concavo-plana, in medio costa unica rotundata instructa, apertura trigona.

La forma di questa conchiglia, quantunque rotta ai margini, sembra che debba essere quasi romboidale, e quindi somigliante alla C. lanceolatc, ma piu ristretta alla parte anteriore, in modo che sembra piu allungata e retta; la regione dorsale è carinata in tutta la sua lunghezza, e sporgente alla parte anteriore; la carena, quantunque ben prominente, non presenta uno spigolo acuto ma rotondato; dall'mno e l'altro lato di essa vi ha una costola ben rilevata, lineare ed appianata; tra essa e la carena ve ne ha un' altra più stretta, ben distinta, elre si estende solamente sino alla metà della conchiglia; la superficie della valva dorsale presentasi striata con molta regolaritì; le strie dall'mo e dall'altro lato della carena mediana sono rette ma disposte obliquamente in rapporto alla carena stessa, in ruodo che su di essa s'incontrano quasi ad angolo retto; la valva ventrale è un po' concava, e presenta nel mezzo una

(1) Recentemente ho raccolto un esemplare di questa specie nelle marne gialle della contrada Pietrazza. 
larga costola poco convessa, con leggiere strie longitudinali; la regione posteriore è rotta, per lo che la conformazione dello sprone ci è sconosciuta; la forma dell'apertura ì triangolare.

Rapporti e differenze. - La nuova specie testè descritta è molto affine alla $C$. lanceolata, dalla quale differisce perchè sembra piu ristretta alla parte anteriore per la carena più distinta, e sopratutto per la conformazione delle costole laterali, che sono rette, ugualmente larghe in tutta la loro lunghezza, ben distinte ed appianate alla parte superiore.

Dimensioni. - L' unico esemplare che possiedo è rotto sui margini, e quindi non conosco la sua larghezza originaria.

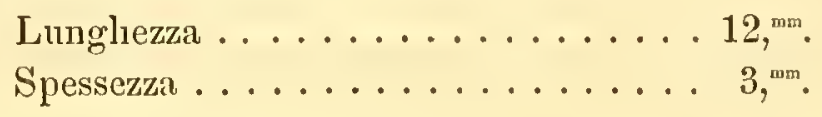

Giacimento. - Calcare F.

Localiti. - Scoppo.

Genere Creseis Rang. 1828.

7. Sp. Creseís striata Rang.

Fig. $13 a, 13 b$.

Sinonimia.

1829. Creseis striata. . Rang. Ann. d. Sc. nat. 13, pag. 315, tom. XVII, fig. 3. 1835-43. Cleodora striata . Lamk. 2. ediz. VII, jag. 433.

1835-42. Crescis striata . D'Orb. Voy., pag. 122, tav. VIII, fig. 23-25.

1840. " " . Cantraine. Malacolog. medit., pag. 32, tav. I, fig. 10.

1843. " sulcata. . Benoit. Ricerche malacologiche, pag. 6 .

1844. $\quad$ striata . Philippi. Enumeratio moll. Sicil., vol. II, pag. 72.

Caratteri. - C. testa clongata, arcuata, compressiuscula fragilissima, inferne vix acuta, annulatim striata; apertura simplici ovato-rotundata. (Cantraine).

Riferisco dubbiosamente a questa specie tre esemplari di Crescis, che sono in cattivissimo stato di conservazione, e non mi permeltono quindi di dare ampia descrizione della specie; anzi lıo dovuto ricorrere al sig. Cantraine per la diagnosi latina. Gli esemplari in discorso sono veli modelli marnosi, che conselvano tuttavia taluni frammenti della fragilissima conchiglia che li ha prodotti, la quale per la forma, per l'estrema fragiliti, e per la natura delle strie trasversali sembria non si discosti dalla specie vivente.

Rapporti e differenze. - La Creseis striata è ben distinta dalle altre specie per le strie trasversali.

Giacimento. - Abita l'oceno atlantico, il mare delle Indie ed il Mediterraneo. Giace fossile nelle marne giallastre.

Localitù. - Trapani presso Messina. 


\title{
FAMIGLA. - SPIRIALIDI (Spirialid(e). Chenu.
}

\author{
Genere Spirialis Souleyet 1840.
}

\section{Sp. Spirialis stenogyra Phil.}

Fig. 11.

Sinonimia.

1844. Scaea stenogyra ... Philippi. Enumerat. moll. Sic., vol. II, p. 164, tav. XXV, fig. 20.

1855. Limacina stenogyra. Pietet. Traité de paléontol., tom. III, p. 317, tav. LXX, fig. 13.

1859. Spirialis stenogyra. Chenu. Manuel de Conch., vol. I, pag. 113, fig. 499.

1862.

$" \quad$. Seguenza. Notizie succinte, ecc., pag. 30.

Caratteri. - S. testa conoidea, obtusa, tenuissima, lavissima; anfractibus rotundatis, sutura profunda divisis, latioribus quam altis, ultimo maximo, ventricoso; apertura spiram suboquante; columella recta, perpendiculari, reflexa, margine reflexo umbilicum formante.

Conchiglia ovato-conica, coll'apice ottuso, fragile molto perchè tenuissima, colla superficie levigata; costituita da sei o sette anfratíi convessi rotondati e disgiunti da suture profonde; gli anfratti superiori sono piì larghi che alti, l'ultimo è molto grande in rapporto agli altri, e di forma globosa. L'apertura è poco meno lunga della spira; il labbro forma, col penultimo anfratto, un angolo acuto, e la columella un angolo ottuso; la columella è retta, perpendicolare, e formata dalla continuazione dell'asse della conchiglia; il margine che la costituisce è ripiegato, e forma nu ombelico; la sua lunghezza è circa metì di quella dell' apertura.

Rapporti e differenze. - La Spirialis stenogyra, per la sua spira elevata, si avvicina alla S. bulimoides D'Orb., e molto più alla S. australis Eydoux. Dalla prima si distingne per gli anfratti molto convessi, per l'apice ottuso, e pel grande sviluppo dell'ultimo anfratto; dalla seconda pel numero minore di giri di spira, per l'angolo aento che fa il labro col penultimo anfratto, per la forma ventricosa dell' ultimo anfratto.

$$
\begin{aligned}
& \text { Dinensioni. - Lunghezza . . . . . . . . . . . . . } 1,{ }^{\mathrm{mm}} 7 \text {. } \\
& \text { Larghezza . . . . . . . . . . . } 1{ }^{{ }^{\mathrm{m}} 1} \\
& \text { Giacimento. - Vivente nel Mediterraneo. } \\
& \text { Fossile nei terreni quaternarii Palermo, Pezzo (Philippi), } \\
& \text { Messina (Seguenza). }
\end{aligned}
$$

Localita. - Milazzo. 


\section{Sp. Spirialis globulosa, Seg.}

Fig. 12.

Carntteri. - S. testa brevi conico-globosa, obtusa, tenuissima, lcevissima; anfractibus rotundatis, sutura profindissima divisis, latissimis, et brevissimis, ultimo magno, inflato; apertura spiram sub-excedente; columella subrecta; umbilico suborbiculato majusculo.

Conchiglia breve, a spira poco elevata, e percio di forma quasi globosa alla parte inferiore, conica ed ottusa superiormente, molto fragile perchè tenuissima, colla superficie levigata, costituita da sei anfratti convessi, rotondati e disgiunti da suture profonde e quasi scanalate; gli anfratti superiori sono molto bassi in rapporto alla loro larghezza; l'nltimo è grande e ventricoso. L'apertura è un po' più lunga della spira; il labro forma un angolo quasi retto col penultimo anfratto; la columella è. breve, retta alla parte inferiore, e costituisce un ombelico ben distinto e circolare.

Rapporti e differenze. - La mova specie descritta ì distintissima dalla S. stenogyra Phil. per la sua brevitì e per l'ombelico circolare, na essa è molto più affine alla S. vertricosa Eydoux, la quale si distingue dalla mia per la spira meno sporgente e per l' ultimo anfratto molto piì grande e più rigonfio.

$$
\begin{aligned}
& \text { Dimensioni. - Lunghezza . . . . . . . . . . . . . . . } 1,{ }^{\mathrm{mm} 3}
\end{aligned}
$$

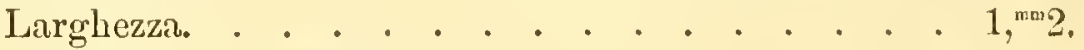

$$
\begin{aligned}
& \text { Giacimento. - Calcare F. e marne giallastre E. } \\
& \text { Localiti. - Pagliarino (nel calcare). } \\
& \text { Rometta, S. Filippo, Trapani (nelle marne). }
\end{aligned}
$$


$-15-$

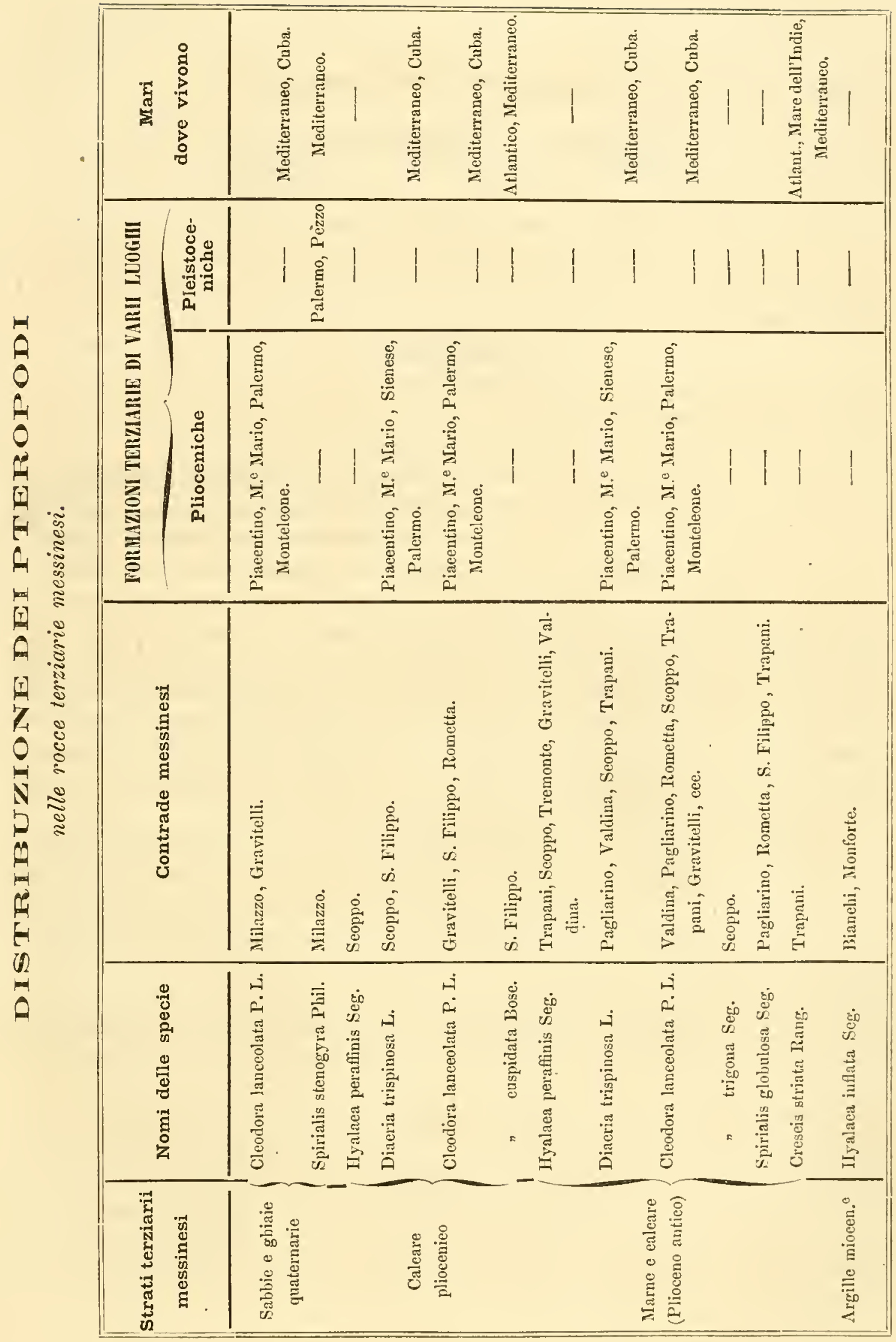




\section{PARTESEOONDA.}

\section{Eteropodi}

\footnotetext{
C'est life par la que la paléontologie est une branche de la zoologie. et que c'est des méthodes de refte science qu'elle dolt s'inspirer. Son but essentiel est l'étude des rapports zoologiques qui existent entre les animaux fossiles, et entre ceux-ci et les vivants........

F. J. Pictet. Traile de paléontologie. Tom. 1. Consid. gènérales, p. 코.
}

Se pei caratteri della loro organizzazione gli Eteropodi sono ben distinti dalla classe dei Pteropodi, essi purnondimeno se ne approssimano grandemente per le abitudini sociali, crepuscolari e pelagiche, nonchè per la estrema fragilitì delle loro conchiglie, allorquando ne sono fornite. Infatti, al par delle Jalee e delle Cleodore, essi ingombrano sovente la superficie dei mari caldi e temperati, formando immense societi, che vengono trasportate dalle correnti.

Nella natura vivente la classe degli Eteropodi, grazie alle numerose e recenti scoperte, appresta oggidi al nostro studio considerevole numero di generi e di specio variatissime; ma gli strati sedimentarii delle epoche preeedenti non lanno conservato i residni delle specie che probabilmente popolavano quei mari, e quindi la paleontologia non vanta che pochi e rarissimi acquisti intorno a questa importante classe dei mollnschi. Un tal fatto, che sembrerebbe a prima ginnta ecceziouale, e clie potrebbe indurre a credere alla mancanza di tali esseri nelle epoche precedenti alla nostra, riceve compiuta spiegazione collo studio dei caratteri e delle abitudini degli Eteropodi viventi.

Tali molluschi infatti per la maggior parte sono sforniti di conchiglia, e quindi i sedimenti marini non possono conservare veruma parte del loro molle corpo; altri sono provvisti d'una conchiglia vitrea fragilissima che si distrugge colla piì grande facilitì, sopratutto per la vita pelagiea di tali esseri, clıe li espone nelle tempeste ad essere rigettati sulle spiagge, dove i marosi agitandoli continhamente su per le ghiaie. operano in hreve tempo la distruzione e del molle animale e della fragilissima conchiglia. 
È per questo che riandando da capo a fondo le opera di paleontologia generale e i trattati speciali dei molluschi fossili di tutte le epoche, non s'incontra quasi mai la descrizione d'un Eteropodo. La paleontologia infatti, ne' snoi immensi odierni progressi, non conosee tuttavia negli strati sedimentarii altri rappresentanti della classe degli Eteropodi se non la Carinaria Mugardi Bellardi, scoperta nella formazione miocenica di 'Torino, la Atlanta Lamenonii che il prof. O. G. Costa scopriva nella marna argillosa bigia di Notaresco, ed il dubbio Ditaxopus del Rafinesque scoperto per una sola volta nelle psammiti di transizione d'America (1); tutti gli altri generi e specie sono solamente riconosciute nella natura vivente.

Tra tanta raritì di Eteropodi fossili credo che debba riuscire importante la descrizione di tre specie raccolte nelle rocce terziarie messinesi, due delle quali spettano al genere Janthina, noto soltanto per le specie viventi.

Di questi tre fossili uno fu raccolto dal sig. Otto nelle rocee messinesi, e descritto dal Philippi colla dubbia denominazione di Cyclostoma delicatum? La conchiglia cosi nominata fu poscia, nelle mie Ricerche intorno ai terreni terziarii del distretto di Messina, dubbiosamente riferita al genere Janthina; e quindi mi sono grado grado confermato sempre meglio in tale idea, ed ormai non dubito punto che tal fossile appartenga al genere suddetto.

Oltre questa specie, recentemente ho raccolto una nuova Janthina nel calcare della contrada Scirpi insieme a copioso numero di Brachiopodi.

Finalmente il terzo Eteropodo, del quale parlerò, è una nuova specie appartenente al fragilissimo genere Carinaria, che recentemente ho raccolto nelle marne giallastre della contrada Trapani.

La grande relazione che esiste tra i Pteropodi e gli Eteropodi, ed il piccol nu'mero di quest' ultimi, mi hamo fatto credere convenerole di descriverli insieme ai primi. E appunto per questo che ho divisato di pubblicare insieme alla monografia dei Pteropodi fossili del Messinese, quella più breve, ma forse più importante, degli Eteropodi che giacciono nei medesimi terreni.

(1) Ho trascurato di aceennare qui la faniglia dei Bellerofontidi (eostituita dai generi Bellerophon, Bucania, Porcellia che spettano all' epoea paleozoica', e Bellerophina della formazione eretacea), la quale essendo, di affinità zoologiche dubbiissime, è stata da taluni scrittori riposta nella classe degli Eteropodi. Sono di tale avviso il L'Orbigny, il Deslaryes, il Bronn; ma, a dire il vero, una tale opinione è contrariata dalle vedute di altri sommi scrittori, che ripongono tali esseri or tra i Cefalopodi ed or fra i Gasteropodi.

Il D'Orbigny stesso adduce valevoli ragioni pel ravvicinamento di questi fossili ai Cefalopodi; opinione segníta da Ferrusac, da Defrance, da Mae Coy, da Chenu.

Il Blainville li ba riposti presso le Bulle, il Fleming li ravvieina agli Acteon, il Koninek li deserive nella famiglia dei Fissurellidi ravricinandoli alle Linarginule, c tale opimione viene seguita dal Pietet. 


\title{
CLASSE. - ETEROPODI (Heteropoda) Lamk. 1819. \\ Nucleobranchiata Blainville, 1824.
}

\author{
FAIIGLIA. - JANTINIDI (Jantlince) Lamk. 1812.
}

Gen. Janthina, Bolten 1798.

\section{Sp. Janthina primigenia Seg.}

Fig. $1 a, 1 b$.

Caratteri. - J. testa tenui, subumbilicata?, depressa, rotundata; lineis elevatis |longitudinalibus flexuosis eleganter ornata; spira brevissima, apice rotundato; anfractibus planatis, suturis impressis disjunctis, ultimo maximo-inflato, apertura magna subtriangulari; columella recta producta.

Conchiglia sottile, molto depressa ed ottusa, anzi arrotondata alla parte superiore, in modo che la spira è pochissimo sporgente, e formata da quattro anfratti, dei quali i superiori sono piccoli, appianati e clisgimuti da suture impresse; l'ultimo è grandissimo, tanto che costituisce quasi da sè solo tutta la conchiglia, presso a poco come nell'Helix aperta Born.; la superficie è ornata da linee di accrescimento sottilissime, rilevate, flessuosamente oblique, indicando che il margine dell'apertura alla sua parte più esterna dovea presentare una leggera sintositì, e così ancora in vicinanza dell' estremità inferiore della columella, dove le strie formano un distinto ripiegamento; l'apertura ingombra della roccia calcare, dalla quale è stata tratta la conchiglia, non mi è ben nota nei suoi particolari, pur nondimeno si può asserire che essa è grande e quasi triangolare, che la conchiglia alla base sembra portare una depressione che la rende quasi ombelicata, e che la columella è retta e sporgente presso a poco come nella $J$. bicolor Menke.

Rapporti e differenze. - Dai caratteri qui sopra indicati e dalle figure, si deve restar certissimi intorno alla definizione generica di tal conchiglia, la quale d'altronde ì distintissima dalle specie viventi sinora solamente conosciute. Infatti sembra avere la mia nuova specie qualehe somiglianza colla $J$. bicolor; ma la grunde breviti della spira, lo sviluppo e la convessità dell'ultimo anfratto, le linee di accrescimento molto sottili e rilevate ne la distinguono eminentemente.

Dimensioni. - Lunghezza della conchiglia . . . . . . 17, 1 , $^{\text {mu! }}$ Larghezza . . . . . . . . . . . 19,

Giacimento. - Nel calcare dello strato F.

Localita. - Scirpi presso Messina. 


\section{Sp. Janthina delicata Seg.}

Fig. $2 a, 2 b, 2 c, 3$.

sinonimia.

1844. Cyclostoma? delicatum Phil. Enumeratio moll. Sic. volume II, pag. 222, tavola XXVIII, fig. 3.

1862. Janthina? delicata Seguenza. Notizie succinte, ece,, pag. 17.

Caratteri. - J. testa tenuissima late umbilicata, lineis elcvatis longitudinalibus, transversisque temuissimis reticulata; spira prominente, anfractibus teretibus, sutura profunda divisis, celeriter crescentibus, ultimo maximo; apertura magna ovatosubromboidali, spiram fere triplo subøquante; labio columellare subrecto, longe prominente; peristomate simplici.

Conchiglia sottilissima fornita d' un largo ombelico, ed ornata da eleganti strie sottili ed elevate, longitndinali e trasversali, che sono presso a poco tutte eguali; la spirale è abbastanza sporgente, e costituita da cinque o sei anfratti molto convessi e disgiunti da profonde suture; essa cresce rapidamente, e l'ultimo giro è molto grande e gibboso in rapporto agli altri; l' apertura ì anch' essa grande, di forma ovatoromboidale, perchè il margine esterno, che è semplice, forma un angolo acuto all'incontro col penultimo anfratto, ed un altro angolo al punto di riunione col margine columellare, il quale forma un angolo ottuso col penultimo anfratto; la lunghezza dell'apertura è quasi tripla della spira; la columella quasi retta (forse esattamente retta negli esemplari intierissimi che mi mancano) e molto sporgente.

Oltre la forma testè descritta riferiseo a questa specie talmi esemplari che convengono esattamente nella forma e nelle strie longitudinali coll'individuo rappresentato dalle fig. 2; ma che se ne allontanano per le strie trasversali, le quali, invece di essere ravvicinate ed equidistanti, sono rare, più distinte, ed a distanze disuguali, come nell' esemplare rotto rappresentato dalla figura 3 ; ma tale scultura fia graduato passaggio, per varii individui, a quella della forma tipica descritta.

Siffatta varieta ho proposto ehe si dicesse $J$. subangulosa, perehè lit ove sono le strie straversali la conchiglia forma una leggerissima piega, che costituisce un' angolositi ottusissima, di maniera che gli anfratti sembrano quasi angolosi.

Rapporti c differenze. - Il Philippi descriveva questa conchiglia nella categoria generiea delle Ciclostome; ma facea ciò con molta riserva. Segnava con punto interrogativo il nome generico, e soggimngeva in fine della descrizione: Societas testarum marinarum etiam hane specien marinam, non terrestrem, fuisse suadet.

Tale considerazione fa vedere chiaramente con quanta incertezza il Philippi riponeva tale conchiglia tra le Ciclostome.

Io faecio considerare che la figura data dal Philippi mostra chiaramente che i margini dell'apertura dell' esemplare da lui disegnato (elie probabilmente dovea essere il più completo tra quanti se ne procurò) crano verisimilmente corrosi, e furono 
dall' antore ristaurati nel disegno; quindi la columella non si mostra retta e sporgente come nell'esemplare da me figurato; l'apertura, lungi di essere angolosa, ¿̀ ovato-ellittica; e percio earatteri di tanta importanza, deformati e contraffatti in tal modo, hanno indotto lo scopritore della specie in discorso a riunirla dubbiosamente alle Ciclostome.

Dallo studio dei poehi esemplari che possiedo della Cyclostoma? delicatum Phil. sembrami indubitato ehe essa si appartenga al genere Janthina. Infatti, eselnsa la possibilità che essa sia appartenuta ad un molluseo terrestre, e ciò per le ragioni stesse addotte dal suo scopritore, e molto pii per la conformazione dell' apertura e della columella, e pel totale difetto di conchiglie terrestri nelle rocee messinesi, essa spettar deve necessariamente ad un mollusco pelagico, e tale ce la annuncia la sna grande sottigliezza. Avendo riguardo poi alle ravvicinatissime strie longitudinali ehe scorrono flessuose ed oblique sugli anfratti, alla conformazione del labbro columellare ed al grande sviluppo dell' ultimo anfratto, sembrami indubitato che tal conchiglia debba riporsi nel genere Janthina.

Dimensioni. - Lungliezza dell' esemplare rappresentato dalle figure $2 \quad 8,{ }^{\mathrm{mm}} 4$.

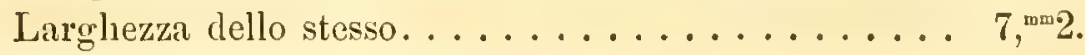

Giacimento. - Nel ealeare dello strato F.

Localitì. - Specie molto rara della contrada Gravitelli e Scoppo.

\section{FAMIGLIA. - FIROLIDI '(Firolidae).}

Gen. Carinaria. Lamarck. 1801

\section{Sp. Carinaria peloritana Seg.}

Fig. 4.

Caratteri. - C. testa elevata, conico-compressa, tenuissima, transversim undulatoplicata, dorso angulato, lamella elevata carinata, apice minimo intorto supra marginem posticum inclinatum; apertura lanceolata, postice rotundata.

Conchiglia molto sottile, di forma conico-elevata, compressa, trasversalmente ondulato-pieghettata, talume delle pieglie circondano tutta la conchiglia, altre intermiste a queste hanno origine dalla regione della carena, e si estendono più o meno verso la regione posteriore; il dorso è angolato, e lungo lo spigolo che esso forma si eleva una lamina stretta costitnente una carena, che sembra sfornita di pieghe; l'apice è piccolo, avvolto ed inclinato sul margine posteriore, l'apertura lanceolata, rotondata dal lato posteriore.

Rapporti e differenze. - La nuova specie testè descritta è molto affine alla C. mediterranea P. e L., dalla quale sembra differire per essere di forma piì elevata, e perehè probabilmente la lamina della carena è sfornita di pieghe. In verità l'esem- 
plare unico che possiedo, e che figura nella mia tavola, trovasi in istato di conservazione troppo cattivo, da permettere compintamente lo studio di tutti $\mathrm{i}$ caratter $\mathrm{i}$ specifici. La conchiglia in gran parte è distrntta, e vi rimane soltanto il modello interno costituito dalla roccia marnosa; la lamina della carena che in esso manca inticramente, mi fu dato osservarla nella marna stessa, studiando l'impronta esterna lasciata da un altro individno della medesima specie; è perciò che nella figura 4 ho ricordato tale parte della conchiglia con una semplice linea, quantunque nell'esemplare figurato tale lamina mancasse del tutto (1).

L' esemplare figurato ha le seguenti

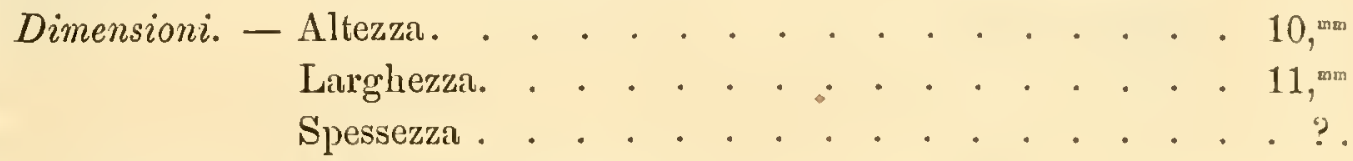

Giacimento. - Marne giallastre E.

Localita. - Trapani presso Messina.

\section{DISTRIBUZIONE DEGLI ETEROPODI NEI TERRENI MESSINESI.}

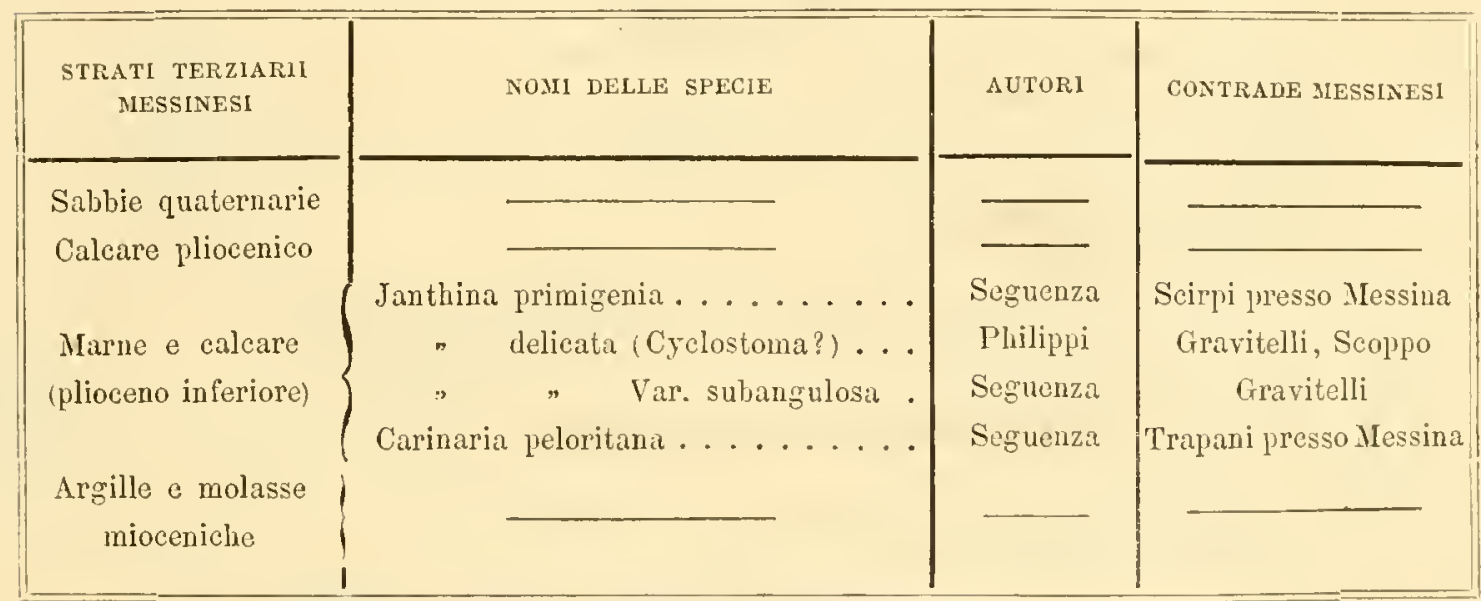

(1) La linea rappresentante la lamina della carena fu traseurata dal litografo. 


\section{SPIEGAZIONE DELLE FIGURE.}

\section{Eteropodi.}

Fig. 1 JANTHINA PRIMIGENIA Seguenza.

" 1 a Un esemplare raccolto alla contrada Scirpi.

$" 1 l$ Lo stesso in posizione diversa.

, 2 JANTHINA DELICATA (Cyclostoma) Philippi.

* 2 a Un individuo intiero raccolto a Gravitelli, disegnato dal lato dorsale e molto ingrandito.

" 28 Lo stesso guardato dalla regione superiore.

" $2 c \quad l l$ medesimo esemplare dal lato dell'apertura.

" 3 JANTIIINA DELICATA (Vur. subangulosa) Seguenza.

" Un esemplare rotto molto ingrandito, proveniente dalla contrada Gravitelli.

4 GariNaria PELORITANa Seguenza.

L' unico esemplare raccolto nella contrada Trapani, rappresentato con ingrandimento considerevole.

\section{PIevoporli.}

Fig. 5 HIALAEA PERAFFiNIS Seguenza.

" 5 a Un esemplare raccolto nel calcare F. a Trapani, guardato lateralmente.

" $5 b$ Lo stesso veduto dal dorso.

- $5 \mathrm{c}$ Regiono ventrale dello stesso.

. 6 IIIALAEA INFLATA Seguenza.

- 6 a Un individuo dell'argilla di Bianchi, in cui si osserva la valva dorsale.

- 66 Un altro delli medesina provenienza veduto dalla regione rentrale.

. $G c$ Un escmplare, raccolto a Mouforte, osservato dalla parte posteriore.

" 7 DIACRIA TRISPINOSA Lesueur.

" 7 a Un individuo guardato dal dorso, mancante degli aculei perchè rotti, l'accolto nello marne giallastre di Trapani.

- 78 ll medesimo esemplare rappresentato dal lato veatrile.

. 8 CLEODORA LANCEOLATA Peron e Lesueur.

- 8 a Un esemplare del calcare F. di Trapani, rappresentato dalla regione ventrale.

. 8 b Lo stesso dal lato dorsale.

. Se Un individuo del calcare F. di Valdina, che costitnisce una variazione a costola mcilitua striata.

"9 CLEODORA TRIGONA Seguenza.

- 9 a L'unico esemplare raccolto nel cilleare F. di Scolpo.

- 98 Lo stesso guartato dal ilorso.

- 10 CLEODORA CUSPIDNTA Bose.

- 10 a l' unico frammento raccolto nel calcare pliocenico di S. Filippo inferiore, rappresentato dal lato ventrale.

" 10 b Lo stesso dal lato dorsale.

" 11 SPIRIALIS STLNOGYRA Phil.

" Un esemplare delle sabbie quatemarie di Milazzo molto ingrandito.

- 12 SPIRIALIS GLORULOSA Seguenza.

" Un individuo delle marne di Rometta molto ingrandito.

" $1:$ CRESEIS STRLTA Rang.

- 13 a Un esemplare ingranlito, rotto, proveniente dalla contrida Trapani.

. 136 Un altro frammento della medesima contra. 
Seguenza, "tere Ftw tero the sins
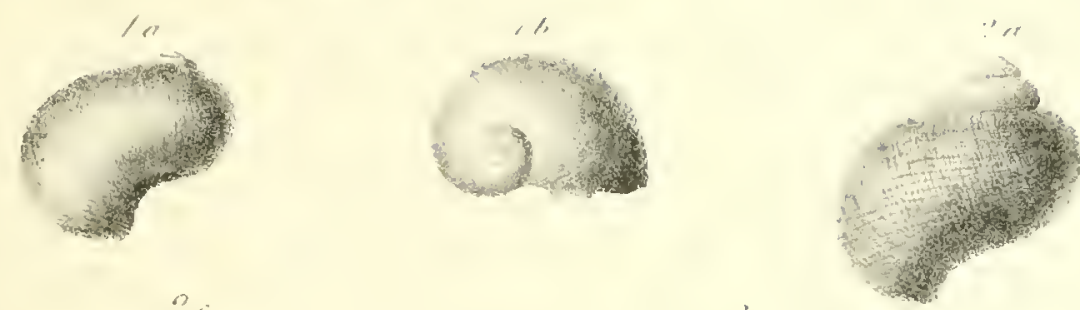

(3)
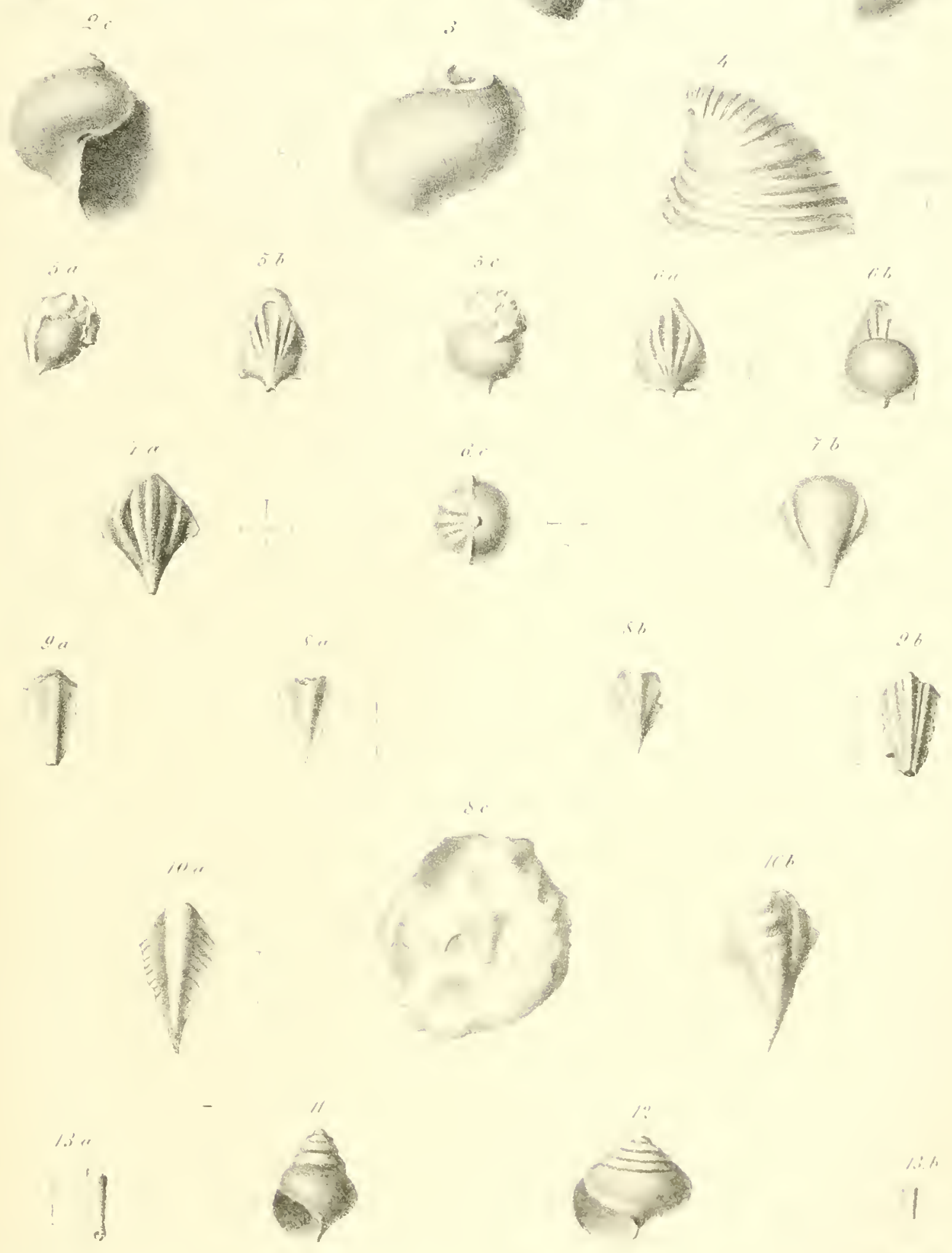

FIE 1 I ETEROPODI H HE 






Carded 



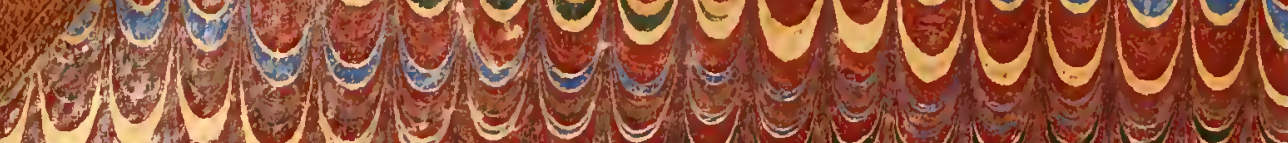

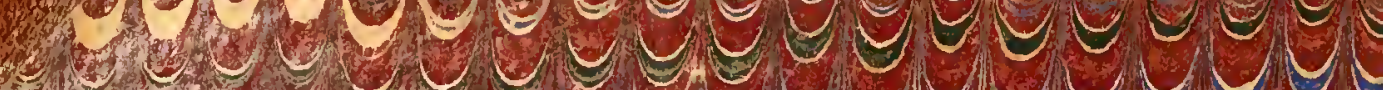

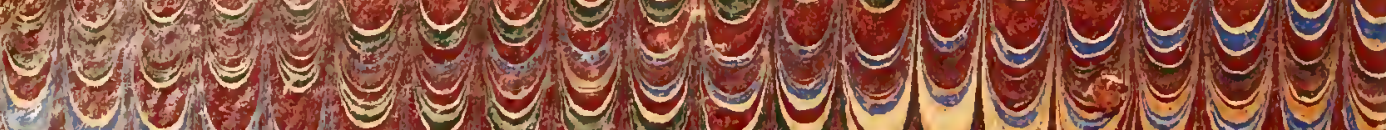

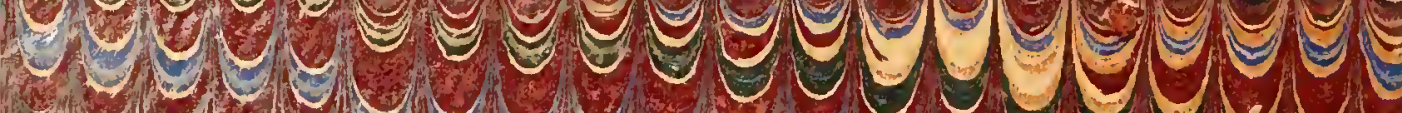
9 y 353

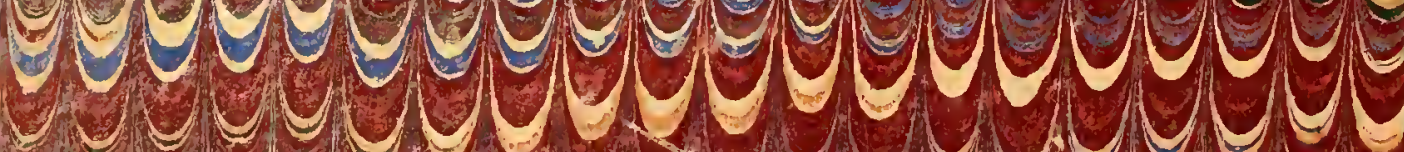
Y y y y

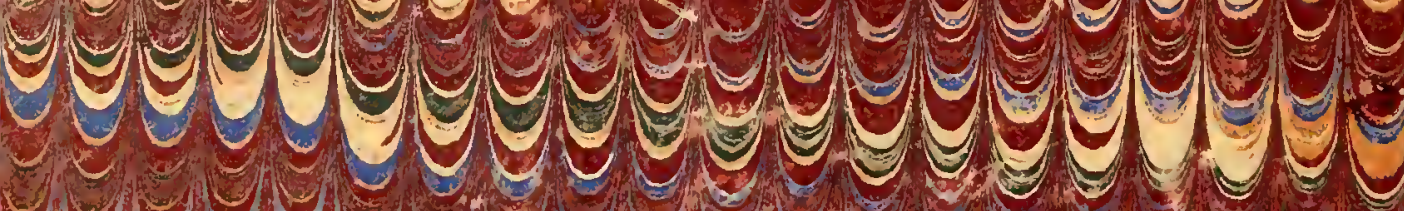

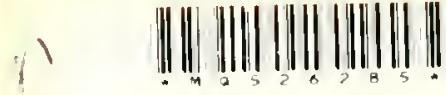

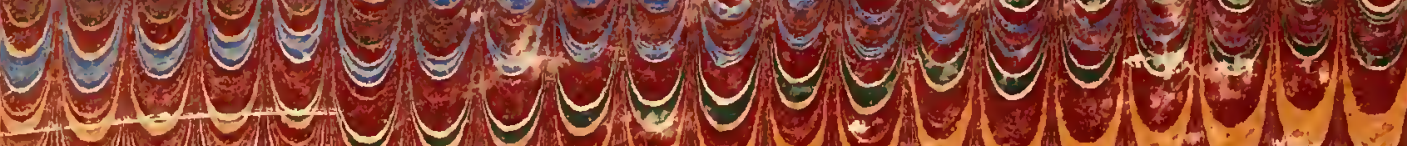

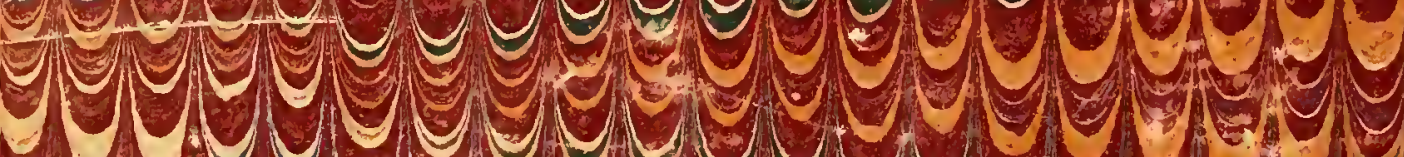

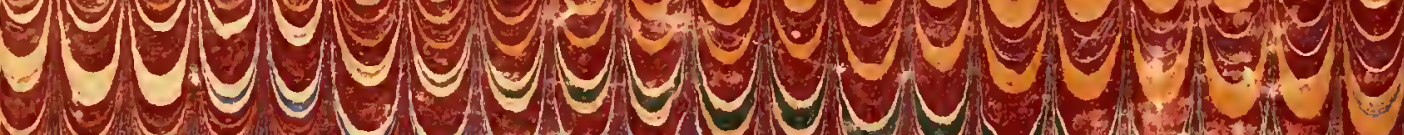

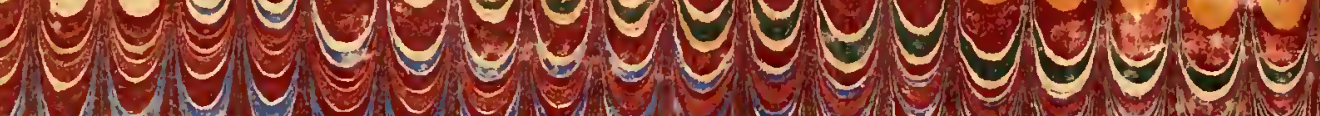

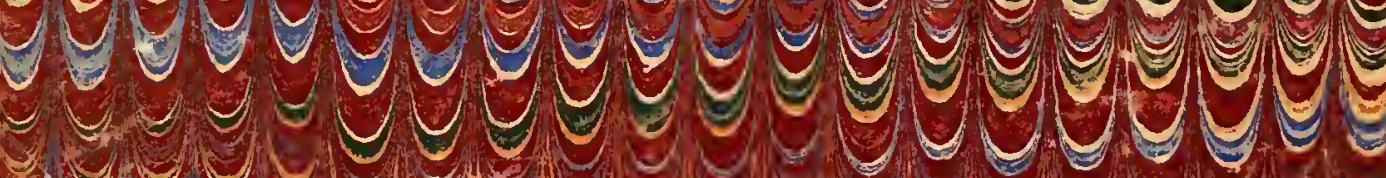
4. y y y y y y y y $9 y 9 y 393$

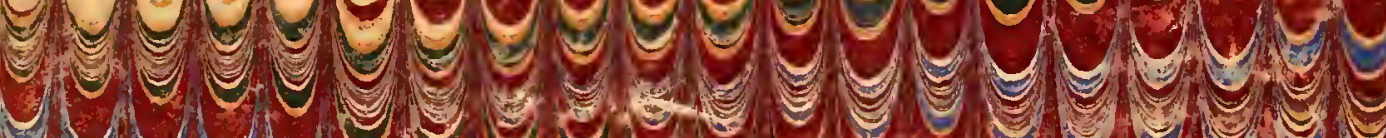

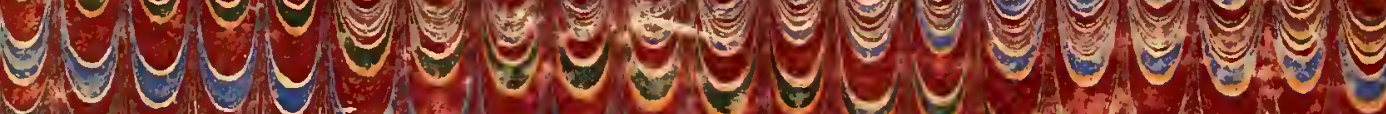

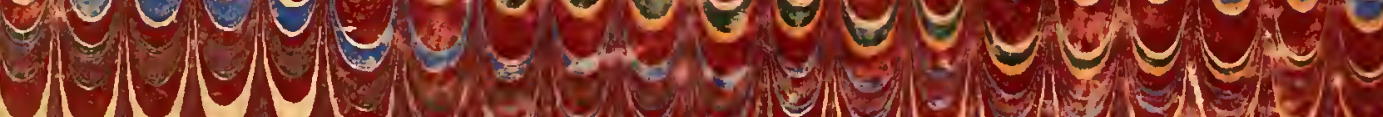
$19 y$ y y 40

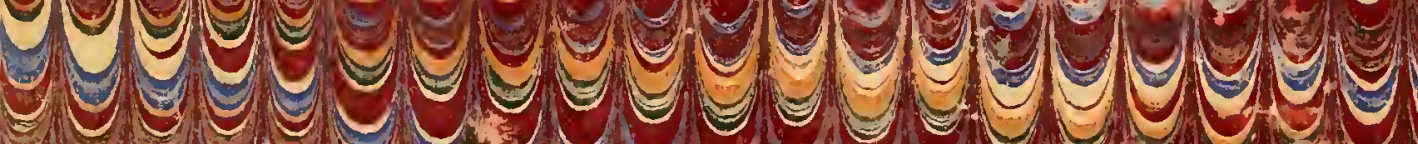

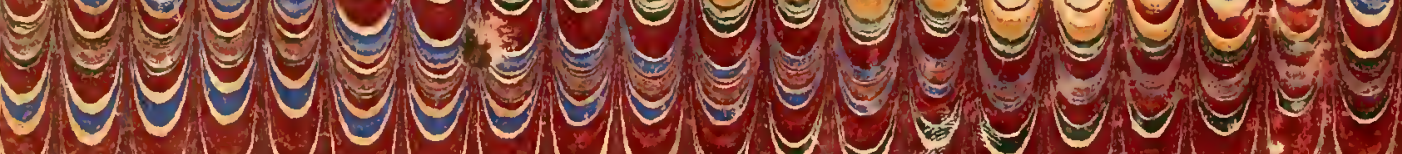

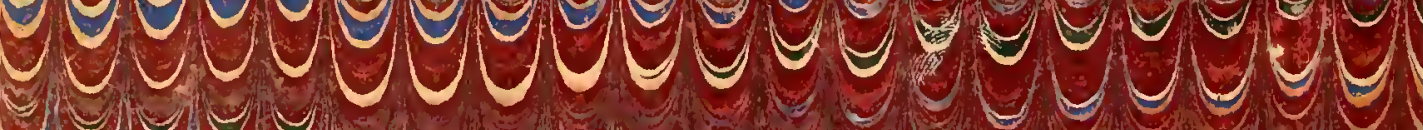
y y y y y y y y (4)

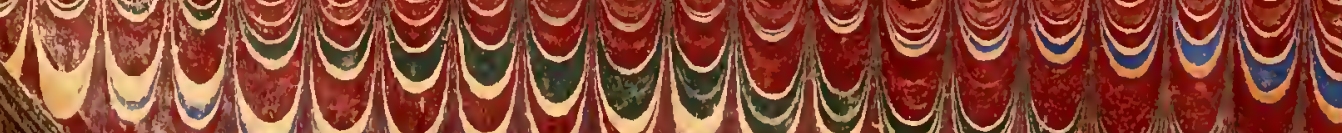

